\title{
Défilé dans le désert : les créateurs de mode vestimentaire noirs de Paris à Agadez
}

\section{Pascale Berloquin-Chassany}

\section{(2) OpenEdition}

Édition électronique

URL : https://journals.openedition.org/remi/5001

DOI : 10.4000/remi.5001

ISSN : $1777-5418$

Éditeur

Université de Poitiers

\section{Édition imprimée}

Date de publication : 1 décembre 2009

Pagination : 191-199

ISBN : 978-2-911627-53-8

ISSN : 0765-0752

\section{Référence électronique}

Pascale Berloquin-Chassany, « Défilé dans le désert : les créateurs de mode vestimentaire noirs de Paris à Agadez », Revue européenne des migrations internationales [En ligne], vol. 25 - n³ | 2009, mis en ligne le 01 décembre 2012, consulté le 15 avril 2022. URL : http://journals.openedition.org/remi/5001 ; DOI : https://doi.org/10.4000/remi.5001 


\section{Note de recherche}

\section{Défilé dans le désert : \\ les créateurs de mode vestimentaire noirs de Paris à Agadez}

\section{Pascale BERLOQUIN-CHASSANY*}

\section{Qu'est-ce qu'il ne faut pas faire pour vendre notre pauvre Afrique!}

(Propos d'une invitée, assistante d'un créateur, FIMA 98)

Paul Gilroy a discuté de l'invisibilité des Noirs dans l'histoire culturelle (2000), invisibilité que j'ai retrouvée au cours de ma thèse de doctorat en examinant le champ de la Mode ${ }^{1}$. Les raisons du monopole occidental sont historiques car les représentations des Noirs ne correspondent pas aux attentes du luxe. Le succès de la vogue ethnique et du sportswear dans les années quatre-vingt-dix corrobore la mise à la marge des créateurs de mode vestimentaire noirs malgré l'intérêt qu'ils suscitent tout à coup. Dans le premier cas, les voici exposés au risque d'un enfermement stylistique empreint de traditions perçues comme ancestrales. Dans le second cas, les créateurs s'inscrivent dans une Mode urbaine, jeune, sportive et musicale qui ne correspond pas aux critères de la haute couture. C'est en effet ce niveau de reconnaissance que visent $88 \%$ des 800 créateurs noirs que j'ai recensés. Ils exercent aux États-Unis, en France, dans la Caraïbe et en Afrique francophone. Afin de comprendre la teneur de leurs discours identitaires et leurs modes de fonctionnement, j'ai compulsé une vingtaine de revues féminines noires ${ }^{2}$ - entre septembre 1998 et septembre 2004 - diffusées dans ces espaces géographiques et travaillé auprès de certains créateurs

\footnotetext{
* Docteur en sociologie, Paris X-Nanterre, enseignante vacataire, membre du MASCIPO (E.H.E.S.S., Paris); pberloquinchassany@gmail.com

1 La majuscule accolée au terme de « mode » renvoie à l'institution spécifiquement vestimentaire et permet de la distinguer du phénomène de mode, pour lequel je conserve le « $\mathrm{m}$ » minuscule, dans la lignée des travaux de Marc Alain Descamps (1979).

2 Dont Ebony (dès 1945), Jet (dès 1951), Essence Magazine (dès 1970) et Sister To Sister (dès 1988) publiées aux États-Unis, Amina (dès 1972), Diva (dès 1999), Miss Ebène (dès 2001), Couleur Métyss (dès 2003), etc. publiées en France et Créola (dès 1992), Pilibo (dès 202), Océana (dès 1999), etc. centrées sur la Caraïbe.
} 
noirs pendant trois ans ${ }^{3}$. Il apparaît que le rapport entre l'assignation - ils sont désignés par les qualificatifs noirs, africains, caribéens ou antillais - et l'instrumentalisation - ils se réapproprient des stéréotypes ou les rejettent, au gré des enjeux situationnels - a pour but de (re)construire une identité noire valorisante ${ }^{4}$. C'est d'ailleurs à cette seule condition que les créateurs noirs peuvent espérer trouver une place dans le marché mondial de la Mode. L'analyse de leurs démarches professionnelles et de leur créativité indique une multiplicité des stratégies. Celles-ci sont tour à tour particularistes et universelles et, généralement, pensées simultanément. Les efforts des créateurs de Mode noirs témoignent non seulement d'une volonté de redessiner une altérité cantonnée au style individuel des créateurs - chacun possède sa propre identité qu'incarne sa griffe - mais également d'un désir d'organisation collective à une échelle internationale. Finalement, l'élite internationale noire compte aujourd'hui des créateurs de Mode noirs qui participent à l'énonciation du contenu de l'identité noire. L'examen de leurs discours renseigne sur la fluctuation de la frontière entre Blancs et Noirs. De l'ordre des représentations, elle peut paraître infranchissable lorsque des arguments essentialisants sont monopolisés - dans des récits journalistiques pour relater une manifestation collective par exemple-, mais elle peut également aller jusqu'à disparaître lorsqu'il s'agit d'être accepté avant tout comme un créateur de Mode. Le contexte d'énonciation doit ainsi être systématiquement rappelé afin de saisir ses enjeux et d'expliquer la diversité apparente de l'identité noire lorsqu'elle est observée dans une perspective mondiale.

Je vais revenir à ce qui a déclenché ma recherche doctorale sur la construction identitaire noire transatlantique et relater le FIMA 98, première édition du Festival International de la Mode Africaine et mes premiers pas sur le terrain. Une amie journaliste en contact avec des rebelles touaregs nigériens m'a annoncée en août 1998 qu'un défilé de Mode était prévu dans le désert nigérien, organisé par un créateur nigérien. Selon ses sources parisiennes et nigériennes, créateurs, politiciens, investisseurs, journalistes français, nord-américains, chinois, japonais et africains devaient s'y rassembler pour l'événement. Et ce, dans un lieu décentré par rapport à Paris, Milan et New York, capitales reconnues de la Mode. La nouvelle m'intriguait probablement pour la même raison qu'elle attirait l'attention de ces invités pressentis, car elle suggérait une rupture dans les représentations de l'autre Africain misérabiliste, héritier d'un passé esclavagiste et/ou colonial. En m'engageant dans cette quête ethnographique multisituée, je me doutais que les modalités d'approche des créateurs noirs devraient s'adapter à leurs situations géographiques et à leurs personnalités afin de multiplier mes chances d'accéder à la dynamique collective projetée par le FIMA. Les boutiques des créateurs de Mode Noirs sont dispersées de ville en ville - à Paris, Dakar, Bamako, Niamey, etc. Je devais les y rejoindre pour percer les codes et les convaincre de m'aider à atteindre Tiguidit dans le désert de l'Air, au nord d'Agadez où devait avoir lieu le festival. Ma démarche nomade se dirigeait vers un village artificiel par son caractère éphémère de l'ordre de la performance - il a été construit de

3 J'ai également réalisé des entretiens avec des créateurs noirs à New York, Washington, Paris, Bamako, Dakar, Niamey, Agadez (Niger).

4 Cette dynamique et les points énoncés ensuite ont fait l'objet d'articles : j'ai analysé la situation des créateurs en Afrique francophone à l'aube des années soixante-dix (2007a), le positionnement de leurs confrères à l'égard de la tendance ethnique (2006) et du succès du terme métissage (2007b) et ma posture à l'égard du travail de Paul Gilroy (2009). 
toutes pièces pour l'événement - et par son atmosphère - les protagonistes d'horizons différents n'avaient a priori aucune raison de se rencontrer en dehors de ce cadre. La féerie esthétique promise par un défilé réunissant des créateurs occidentaux et noirs avait pour objectif d'après son concepteur de prouver au monde, grâce aux médias mobilisés, que les créateurs noirs étaient équivalents à leurs confrères occidentaux, membres de l'élite du luxe. La venue de ces derniers, dans un lieu généralement perçu comme hostile - parce que désertique, situé dans un des pays les plus pauvres de la planète - légitimait tacitement le FIMA. Son caractère élitiste invitait l'ethnologue à prévoir des tenues adéquates, élégantes (talons, jupe), un langage choisi et des arguments pertinents (cooptation, positionnement universitaire, etc.) pour participer à la manifestation. Cette épreuve initiatique sur le terrain en Afrique s'est avérée très urbaine, jusque dans le désert, et impliquait la prise de connaissances de codes à mi-chemin entre un champ de la Mode occidental et un champ élitiste noir. C'est en participant aux festivités que j'ai pu constater l'ampleur de l'enjeu identitaire de cette rencontre imprévisible entre des représentations culturelles diverses aux multiples formes.

\section{À LA RENCONTRE DE L'AUTRE NOIR}

Compte tenu de ma personnalité, de mon parcours et de mon apparence physique de jeune femme blonde aux yeux bleus cerclés de lunettes, une réflexion sur les Noirs me place à première vue à l'extérieur du champ d'investigation. J'ai en effet grandi dans un entourage qui ne comptait pas de Noirs 5 . Des vacances en Côte d'Ivoire en $1994^{6}$ et mes recherches sur les relations diplomatiques d'Haïti lors de ma maîtrise d'histoire ont transformé la peur que j'avais des Noirs en curiosité intellectuelle (Berloquin, 2004 ; Bonacci et al., 2006). Par ailleurs, je n'étais nullement intéressée par la Mode. J'en ignorais tout jusqu'au moment où mon amie journaliste a lancé le pari d'aller vérifier la réalité des préparatifs du FIMA par deux approches et deux personnalités différentes, journalistique et scientifique.

\section{PARIS-NIAMEY}

En 1998, Alphadi, créateur d'origine malienne et nigérienne et instigateur du FIMA est considéré comme une personnalité de la Mode africaine en raison d'une diffusion

5 Durant mon enfance, je n'ai croisé qu'une enfant noire avant mon entrée au lycée. Elle était peu appréciée en raison de son agressivité. Malgré cette barrière, je m'efforçais de jouer avec elle parce que nos pères étaient amis. Sans grand succès.

6 Lors de ce séjour, j'ai été frappée par les connexions qui rattachaient des villageois éloignés de tout à la mondialisation et par leur mobilité à travers le continent. J'ai par exemple croisé Jackson dans un village de pêcheurs ghanéens à proximité de San Pedro en Côte d'Ivoire, surnommé ainsi en référence à Michael Jackson. Est-ce la télévision du village ou le passage régulier de l'élite blanche qui l'explique ? À proximité d'Abidjan, à Assinie, où se trouvent le Club Méditerranée France - et son équivalent italien, j'ai rencontré celui que les gens dans l'unique rue appelaient le Roi des Rastas. Il avait joué ce rôle dans un film en Afrique du Sud et avait conservé sa couronne. Il vendait aux touristes des productions artisanales aux couleurs rastas -vert, rouge et or - aidé de sa femme, originaire de Sarcelles, qui leur proposait des crêpes. Ils répondaient en somme tout à fait aux attentes des touristes européens en quête d'exotisme. 
médiatique importante en France et en Afrique francophone ${ }^{7}$ complétée par un bouche à oreille très actif dans le monde noir parisien. Il tient boutique à Niamey et sa réussite professionnelle lui confère un rôle exemplaire, il est d'ailleurs surnommé Ambassadeur culturel de l'Afrique. En octobre 1998, il organise une conférence de presse à Paris à laquelle j'assiste grâce à la complicité de la secrétaire de l'ambassade du Niger, rencontrée précédemment. Cette aide ne suffit pourtant pas à accéder à Alphadi que je souhaitais persuader de m'inviter au FIMA. Impressionnée par l'assurance ostentatoire des journalistes et des attachés de presse occidentaux, je n'arrive guère à lui adresser la parole. Je devine ce milieu fermé, régi par des règles propres de cooptation. Il me faut donc me débrouiller pour obtenir des recommandations de personnalités reconnues comme importantes aux yeux d'Alphadi. J'avais déjà rencontré le mois précédent des créateurs noirs à Paris en piochant leurs adresses dans le numéro de la Revue Noire ${ }^{8}$ qui leur était consacré. Franchir le pas de leurs boutiques m'avait permis d'obtenir les premiers entretiens sur le mode de l'improvisation, mais aucun n'était proche d'Alphadi ou invité au FIMA. Leur cordialité parisienne m'a toutefois encouragée à tenter ma chance en Afrique de l'Ouest auprès de leurs confrères signalés dans le dossier de presse du festival distribué lors de la conférence de presse. À la fin du mois d'octobre, je me rends donc à Dakar au Sénégal, à Ouagadougou au Burkina Faso, à Bamako au Mali, et récolte les récits de parcours professionnels des créateurs dans leurs boutiques de Mode. Ces derniers me promettent leur soutien pour approcher Alphadi dont ils me brossent un portrait engageant. Selon leurs dires, il serait favorable à ma requête. Ce n'est qu'une fois sur place que je réaliserai que l'entrée au FIMA était une entreprise difficile puisque parmi tous ceux qui se disaient invités bien peu s'y rendirent.

J'arrive à Niamey le vendredi 6 novembre et rencontre moult candidats à un entretien avec Alphadi dans sa boutique. Est-ce en raison de mon apparence physique ? Toujours est-il que nul ne m'interroge sur le motif de ma visite et que le créateur me reçoit en priorité dans son bureau. Avec le recul, je ne sais ce qui de mon projet, de mes recommandations ou de ses besoins organisationnels l'emporta dans sa décision de m'accorder sa confiance. Entre deux appels à l'autre bout du monde, il me nomme Responsable du suivi des créateurs africains pour toute la durée du FIMA. L'étonnant réside dans l'image qu'il m'assigne au comité d'organisation, me voici : étudiante-styliste. Alphadi émet tout de même par avance une réserve à mon enthousiasme : "tu vas voir, c'est un cadeau empoisonné, les couturiers sont des stars insupportables ».

Sous couvert de mon étiquette de styliste, je déambule librement dans l'atelier entre les artisans, les journalistes ${ }^{9}$, les collaborateurs d'Alphadi et les coopérants qui me proposent un hébergement avec fer à repasser. Le détail a son importance, je vivais chez

7 Principalement noire - revues, émissions de télévision, radio, etc.

8 Décembre 1997 - janvier 1998, Paris.

9 La télévision nigérienne, en collaboration avec celles du Burkina Faso et de France 3 Limoges, visite l'atelier. Je rencontre ainsi un journaliste qui m'aidera à Agadez et me fournira, de retour à Paris, une cassette vidéo du FIMA. 
un artisan bijoutier targui rencontré lors de mon périple ${ }^{10}$ et ne pouvais dans ces conditions soigner ma tenue vestimentaire et correspondre au luxe affiché dans la boutique. Relookée, je quête des travaux afin d'intégrer l'équipe avec plus ou moins de succès. Toutefois, je suis au cœur de l'action, participe aux transactions financières, aux tracas logistiques, je me rends utile autant que possible.

Dimanche 8 novembre, le temps s'accélère brusquement. Les premiers créateurs en provenance du Mali et de la Côte d'Ivoire atterrissent à Niamey. Je profite de la lenteur des formalités de douanes pour nouer des complicités avec eux et la trésorière de la FAC, Fédération Africaine des Créateurs de Mode et du Design dont j'apprends alors l'existence internationale. Comme avec Alphadi, ma position d'étudiante socio-anthropologue disparait rapidement de leurs esprits en raison du contexte festivalier et de l'aide logistique que je leur apporte. C'est ainsi que j'assiste aux conversations et j'observe en silence, jusqu'au départ pour les portes du désert. Ce moment se produit dans la nuit du mardi 10 au mercredi 11 novembre, marqué par l'impératif de calmer les créateurs devant l'attente prolongée des avions. Après un vol sans encombre pour Agadez, la disparition des accessoires d'une créatrice namibienne me conduit à dialoguer avec les anglophones du groupe, ce qui fait de moi leur interprète. Cette complicité s'avère fort utile dès l'arrivée dans la ville d'Agadez. La question de l'hébergement tourne au drame, et les créateurs, éreintés, trouvent quelqu'un à qui se plaindre, convaincus à juste titre que leurs griefs seront transmis à Alphadi. Me voici de part et d'autre considérée comme une médiatrice, position qui a le mérite de favoriser la récolte de propos spontanés dans ces moments de tension.

\section{AUX PORTES DU DÉSERT}

Les talons et les petites robes sont prohibés en raison du sable et d'une religion pointilleuse sur l'apparence. Il ne me reste qu'à enfiler un jean, à endosser le tee-shirt FIMA - tous deux devenus totalement poussiéreux - et à m'accommoder d'une paire de sandales en plastique achetée en catastrophe au marché d'Agadez. Ma tenue contraste avec celle des invités qui suivent les règles tacites d'un festival de luxe. Pourtant, dans ce contexte, leur respect à mon égard ne dépend pas d'une renommée dans le champ de la Mode comme ce peut être le cas en Occident, mais de ma maitrise de la ville et du programme festivalier. C'est à l'occasion d'un quiproquo que je réalise l'importance d'un autre facteur déterminant pour asseoir ma position : la caution politique. Alors que je m'efforce de calmer les revendications des créateurs originaires d'Afrique, l'arrivée du ministre du Tourisme Rhissa Ag. Boula, ancien chef des rebelles touaregs, transforme ma position. Je l'aborde dès son arrivée afin de savoir s'il sait où trouver celui que je suppose être son ancien compère de lutte qui héberge mon amie journaliste que je souhaite retrouver. À l'évocation du nom d'un partisan d'une cause qui lui était chère, il m'embrasse sur les deux joues tout en me promettant de transmettre mon message. Le déroulement public de cette scène indiqua à tous que je connaissais le ministre et renforça ma crédibilité aux yeux des créateurs en présence et des organisateurs. Le soir, Alphadi me demande de les réunir

10 Avec ses confrères, il était convaincu d'accéder au festival pour y vendre ses bijoux et prévoyait de m'y amener. À tort, aucun d'entre eux n'atteignit Tiguidit, le droit d'entrée pour les artisans étant prohibitif. 
afin qu'ils puissent réfléchir aux difficultés professionnelles auxquelles ils sont confrontées et aux moyens à mettre en œuvre pour les dépasser. Il est occupé en d'autres lieux, les langues se délient et je peux noter sans la moindre critique la teneur de leurs échanges qui portent sur leurs ambitions d'accéder à une visibilité internationale.

Mercredi 11 novembre, des complications logistiques me rapprochent d'Alphadi. Il m'invite à l'accompagner dans le désert pour superviser les opérations. Il doit au préalable régler quelques affaires ce qui me permet d'enregistrer un entretien avec lui en tête à tête. Tout à coup, le dîner organisé pour les créateurs lui revient à l'esprit. Notre trajet s'arrête au restaurant. Les créateurs veillent à ce que mon assiette soit pleine puis vidée de la main droite, dans le respect de la coutume musulmane. Je découvre ainsi les codes de sociabilité qui me permettent de remplir ma double mission, d'universitaire et d'assistante. La journée suivante est intense. Il faut récupérer les collections pour les envoyer dans le désert, récupérer les invités disséminés aux quatre coins de la ville pour les conduire au spectacle de danse de chameaux qui ouvre les festivités. L'équipe organisatrice gère les caprices des uns, recherche les disparus qui visitent les alentours, etc. sans autre moyen de communication que la voiture. La tension éclate lorsque la rumeur se répand que les chauffeurs n'ont pas été payés. Leur refus de reprendre le travail sème un vent de panique. Le transfert des invités vers le désert est imminent. L'arrangement avec les grévistes ne suffit pas, je dois solliciter la préfecture. Ces désagréments laissent entrevoir les imbrications politiques et économiques du FIMA et la position tour à tour puissante et fragile d'Alphadi. Finalement, le convoi s'élance dans le désert sur une route spécialement construite à la sortie d'Agadez. Qui a remarqué le regroupement des familles dans des tentes de fortune à proximité de l'aéroport? Elles ont loué au créateur leurs toits afin qu'il puisse héberger ses invités. Progressivement, le paysage s'assèche, des dunes de sable apparaissent et le décor se fige : des tentes touarègues et marocaines sont regroupées par endroits, la piste en forme de croix d'Agadez délimite l'est du village. La vision est grandiose, l'agitation humaine devient infiniment petite lorsque l'on grimpe sur une dune pour rejoindre l'espace satellite afin d'envoyer des emails en Europe. La nuit, des rangées de tubes de néon plantés dans le sable apportent une atmosphère irréelle. Les Occidentaux sont noyés dans l'immensité du désert, occupés à chercher à manger et à boire. À la nuit tombée, une griotte malienne chante devant les invités qui ont bravé le sable pour la voir. Les autres se roulent gelés dans des couvertures, effrayés parfois par les petites souris blanches qui observent les nouveaux arrivants.

\section{TIGUIDIT : SES DUNES DE SABLE ILLUMINÉES LE TEMPS D'UN DÉFILÉ}

Vendredi 13 novembre, dès le réveil, les créateurs préparent le spectacle du soir dans la cabine d'essayage. Dans ce Back Stage insolite, je circule discrètement et repère trois groupes bien distincts ; les mannequins faméliques - blancs, noirs, chinois - et les créateurs entassés aux côtés des représentants des couturiers européens. Peut-être que de l'harmonie artificielle lors du défilé naîtront des liens entre ces acteurs ? Pour l'instant, chacun s'observe de son entre soi rassurant. À l'autre extrémité du village, des jeunes femmes asiatiques posent aux côtés des militaires qui arborent le kalachnikov. Le site est placé sous haute surveillance. La tempête de sable du matin a laissé la place à un soleil 
écrasant. Les festivaliers conservent toutefois leurs chèches, quand le couturier Kenzo arbore son bob, assis sur un chameau. Le temps semble ralenti. Les journalistes agacés de ne pouvoir transmettre au monde la nouvelle finissent par s'asseoir à l'ombre des tentes. Lorsque le ciel s'obscurcit, les Occidentaux rejoignent les quelques Touaregs qui regardent, bouches bées, le bloc de douze tonnes de glace débarqué de Norvège fondre dans le désert. Ce présent publicitaire du principal sponsor, une entreprise de vodka, est morcelé et creusé en verres emplis ensuite de leur boisson afin de désaltérer les festivaliers ${ }^{11}$. Peu après, 500 personnes affamées attendent le festin. Les chaises sont alignées, tassées autour d'un espace meublé par trois immenses tables encore vides. Le premier chameau ${ }^{12}$ tarde, des serveurs apportent des salades. La cohue se propage comme une traînée de poudre. Des sommités occidentales invitées se ruent sur la table et s'acharnent, la table renversée, à racler les plats dans le sable. Une journaliste AFP me confiera son aventure : " j'ai eu de la chance, j'étais devant le plat, j'ai été la première servie. Et, tout à coup, j'ai vu une fourchette descendre dans mon assiette et piquer ma viande! J'ai tourné la tête, il y avait un Targui, il devait avoir faim, il a continué à m'ôter la viande, j'ai mangé les nouilles qui accompagnaient le plat ». La Secrétaire de l'ambassade du Niger à Paris est consternée : "vous n'avez pas mangé du chameau tout de même, c'est un animal sacré ». L'événement magique, qu'elle avait à cœur d'encourager, semble à ce moment prendre la tournure d'une farce grossière. Ses paroles trahissent l'étonnement devant la transgression d'une règle propre à sa culture quand le mien porte sur la transgression d'une règle de savoirvivre occidental : où est la retenue préconisée devant l'offrande d'un repas ? Tout à coup, le bruit monte et rappelle tout le monde à l'ordre : l'Opéra du désert ouvre trois heures de défilé de Mode. Entre des dunes illuminées, une toile de fond de visages wodaabés ${ }^{13}$ sert d'écran avec la cabine d'où sortent les mannequins. Le spectacle est saisissant. Les tenues d'une trentaine de créateurs africains, occidentaux et chinois circulent avec majesté sur le podium entouré de tapis colorés occupés par les festivaliers. Pourtant, la fête prévue pour clore cette féerie orchestrée par la Princesse du Burundi n'attire personne, chacun retourne à sa tente, comme s'il avait été anesthésié.

À l'aube, aucun des colloques annoncés lors de la conférence de presse n'a lieu. Ceux-ci devaient porter sur les perspectives professionnelles des créateurs et sur le Sida en Afrique. Les journalistes déplorent ouvertement le décalage entre la promesse d'une logique solidaire - qui relève de la santé publique et d'une ambition économique transnationale ${ }^{14}$ - et sa non-réalisation. La majorité des invités est rentrée directement à Paris, Abidjan ou Niamey. L'effervescence s'est dissipée furtivement, s'apparentant dès lors à une parenthèse dans un rythme occidental. Parmi les mannequins d'une grande agence

11 En échange, douze des créateurs africains défilant au FIMA créèrent un vêtement en son honneur et le présentèrent à New York en mai 1999.

12 Extrait du Canardo du 2 novembre 1998, journal satirique nigérien. Ce mets dit des « cinq animaux entrepénétrés » est constitué d'un chameau dont la carcasse est garnie par un bœuf qui aurait avalé un mouton empli à son tour par des poulets.

13 Ethnie peule habitant au nord du Niger, autodénommée.

14 Une table ronde devait rassembler les créateurs de Mode invités et des partenaires potentiels afin de présenter aux journalistes la dynamique de professionnalisation en gestation. 
française venues bénévolement ${ }^{15}$ défiler, une voix s'élève : " on nous a présenté ça comme un défilé pour les pauvres Africains, pour redorer le blason du pays [...] mais il manque totalement de structures humanitaires. On leur a juste apporté : rien. A part quelques babioles à acheter, ils n'ont plus qu'à saliver devant un spectacle qu'ils ne verront même pas ${ }^{16}$. Ses collègues réagissent, sur le chemin du retour. Dans les $4 \mathrm{X} 4$, les cervelles s'agitent : " il faut fonder une association et ramasser des trucs utiles, des cahiers, des stylos par exemple. Oui mais il faut les amener nous-mêmes parce que moi j'ai pas confiance, t'as vu avec le truc pour le cancer ». Cet extrait de mon cahier de terrain suggère de nouveau le contraste entre les attentes des invités occidentaux et la réalité du festival. Le désir de se donner une bonne conscience reste en suspens, faute d'infrastructure. C'est, à mon sens, le contexte qui justifie de telles allégations. Car, lorsqu'un défilé se produit en Europe, le contraste entre le luxe déployé et la misère environnante n'est guère soulevé.

Ce premier terrain m'a enseigné le décalage entre les projections, les possibilités et les moyens mis en œuvre pour y répondre. J'ai constaté que les Occidentaux venus jusqu'à Agadez s'attendent à ce qu'Alphadi leur offre une vitrine du luxe en Afrique, qu'il tienne sa promesse en somme. Et l'équipe responsable de l'organisation est liée personnellement à ce serment. Chacun représente le créateur, le valorise et se met à la disposition des invités afin que le contentement puisse gagner ces derniers. Malgré des désagréments, après coup, les invités purent négocier avec leurs souvenirs c'est-à-dire établir des compromis et prendre position en affirmant : "c'était super ! " Ce type de reconstruction est ressorti très fréquemment de mes entretiens de retour à Paris. Dans le désert, la mauvaise humeur allait bon train. La raison la plus fréquemment invoquée pour expliquer ce changement d'opinion est culturelle : "c'est une culture différente. C'est vrai, le confort dans un pays si pauvre, faut pas s'y attendre [sur place, les mêmes se plaignaient : "ils auraient pu prévoir des couvertures, nous dire qu'il fallait en amener que, la nuit, il faisait froid dans le désert »] et c'était merveilleux ces couleurs, ces tissus, ces chameaux, etc. ». La tolérance énoncée a posteriori dans ces propos indique pourtant une pointe de condescendance qui s'éloigne quelque peu du désir d'Alphadi d'obtenir de la part des Occidentaux une reconnaissance égalitaire.

\section{POUR CONCLURE}

Tour à tour invisible et active, je dois l'observation de ces interactions à la confiance que m'ont témoignée les créateurs de Mode africains. À leur service, j'ai pu comparer leurs comportements en situation de représentation et en contexte privé et vérifier la souplesse de leurs énonciations identitaires, adaptées aux attentes de leur auditoire. J'ai ainsi repéré des éléments révélateurs d'une connaissance des codes et des enjeux du luxe en concordance avec les ambitions élitistes des créateurs de Mode : constitution de réseaux nationaux, continentaux et mondiaux ; maîtrise d'un langage spécifique ;

15 Seuls les mannequins originaires de Côte d'Ivoire et du Niger ont été rémunérés.

16 Notons que tous les soirs, pendant la durée du festival - du 11 au 16 novembre 1998 -, un spectacle est proposé à la population d'Agadez écartée de Tiguidit, comme pour calmer la moindre manifestation de mécontentement. Les bannières du sponsor très présentes sur le site du FIMA, ornent également la scène. 
coexistence d'une animosité concurrentielle et d'une amitié ostentatoire entre eux, etc. Ces différentes pistes m'ont fourni une grille de lecture pour aborder ultérieurement les comptes-rendus médiatiques. Lorsque l'éclairage du podium s'est éteint, je possédais de nombreuses informations. Il me restait à dépasser un étrange sentiment de désillusion. J'étais écœurée par les contrastes ostensibles : l'alcool versé dans un pays musulman, la fascionita japonaise posant aux côtés d'un militaire armé, les biens nourris qui se sont rué sur la nourriture écartant sur leurs passages ceux dont le ventre sonne creux au quotidien. En parallèle, les festivaliers lorsqu'ils étaient regroupés prônaient l'harmonie. Et surtout, malgré la confusion qui régnait, le miracle tant esthétique que symbolique s'était produit. L'objectif proclamé lors des conférences de presse a été atteint, les acteurs du nord se sont mêlés aux acteurs du sud. Concrètement, la distante a persisté, s'est estompée et a resurgi entre eux sous des formes surprenantes. Pour nombre de tenues, il n'était pas possible de repérer l'origine géoculturelle du créateur. Les moyens technologiques les plus sophistiqués étaient proposés aux journalistes quand la misère présumée des Nigériens a été camouflée. C'est bien la magnificence que les invités étaient venus chercher et devaient rapporter chez eux, au Mali, aux États-Unis, en France, en Chine, etc.

\section{Références bibliographiques}

BERLOQUIN-CHASSANY Pascale (2004) Haïti, une démocratie compromise : 1890-1911, Paris, L'Harmattan, $284 \mathrm{p}$.

BERLOQUIN-CHASSANY Pascale (2006) Créateurs de mode vestimentaire africains et labellisation ethnique (France, Antilles Afrique de l'Ouest francophone), Autrepart, 38, pp. 173-189.

BERLOQUIN-CHASSANY Pascale (2007a) Contester la suprématie des dictats occidentaux dans le domaine de l'apparence : l'émergence du créateur en Afrique francophone, in Dominique Veillon et Michèle Ruffat Éds., La Mode des sixties, Paris, Autrement, pp. 98-101.

BERLOQUIN-CHASSANY Pascale et BOIDIN Capucine (2007b) Métis sale ou métis chic. De Misiones (Paraguay) à la Black Atlantic, in Jean-Pierre Castelain, Serge Grüzinski et Carmen Salazar-Sollers Éds., De l'ethnographie à l'histoire. Paris-Madrid-Buenos Aires. Les mondes de Carmen Bernand, Paris, L'Harmattan, pp. 55-72.

BERLOQUIN-CHASSANY Pascale (2009) L'Atlantique noir des créateurs de mode vestimentaire au XXIe siècle, in Carlos Agudelo, Capucine Boidin et Livio Sansone Éds., Autour de l'«Atlantique noir ». Une polyphonie de perspective, Paris, Éditions de l'IHEAL, pp. 73-81.

BONACCI Giulia, BECHACQ Dimitri, BERLOQUIN-CHASSANY Pascale et REY Nicolas Éds. (2006) La Révolution haïtienne au-delà de ses frontières, Paris, Karthala, $233 \mathrm{p}$.

DESCAMPS Marc-Alain (1979) Psychologie de la mode, Vendôme, PUF, 210 p.

GILROY Paul (2000 [1993]) L'Atlantique Noir. Modernité et double conscience, Paris, Éditions Kargo, 298 p. 